\title{
Coherent deeply virtual Compton scattering off ${ }^{3} \mathrm{He}$ and neutron generalized parton distributions
}

\author{
Matteo Rinaldi ${ }^{\mathrm{a}}$ and Sergio Scopetta ${ }^{\mathrm{b}}$ \\ Dipartimento di Fisica e Geologia, Università degli studi di Perugia and INFN sezione di Perugia, \\ via A. Pascoli, 06100 Perugia, Italy
}

\begin{abstract}
It has been recently proposed to study coherent deeply virtual Compton scattering (DVCS) off ${ }^{3} \mathrm{He}$ nuclei to access neutron generalized parton distributions (GPDs). In particular, it has been shown that, in Impulse Approximation (IA) and at low momentum transfer, the sum of the quark helicity conserving GPDs of ${ }^{3} \mathrm{He}, H$ and $E$, is dominated by the neutron contribution. This peculiar result makes the ${ }^{3} \mathrm{He}$ target very promising to access the neutron information. We present here the IA calculation of the spin dependent GPD $\tilde{H}$ of ${ }^{3} \mathrm{He}$. Also for this quantity the neutron contribution is found to be the dominant one, at low momentum transfer. The known forward limit of the IA calculation of $\tilde{H}$, yielding the polarized parton distributions of ${ }^{3} \mathrm{He}$, is correctly recovered. The extraction of the neutron information could be anyway non trivial, so that a procedure, able to take into account the nuclear effects encoded in the IA analysis, is proposed. These calculations, essential for the evaluation of the coherent DVCS cross section asymmetries, which depend on the GPDs $H, E$ and $\tilde{H}$, represent a crucial step for planning possible experiments at Jefferson Lab.
\end{abstract}

Generalized parton distributions (GPDs), introduced in Ref. [1], describe the hadron non perturbative structure in hard exclusive processes. The study of these quantities is very important to understand several open issues of hadrons, such as, e.g., their spin structure. GPDs are related to the total angular momentum of partons inside the hadron; their knowledge, once the helicity quark contribution were subtracted, would make possible to access their orbital angular momentum (OAM) content.

The most promising process for these studies is likely deeply virtual compton scattering (DVCS), i.e., the reaction $e H \longmapsto e^{\prime} H^{\prime} \gamma$ when $Q^{2} \gg M^{2}\left(Q^{2}=-q \cdot q\right.$ is the momentum transfer between the leptons $e$ and $e^{\prime}, \Delta^{2}$ the one between hadrons $H$ and $H^{\prime}$ with momenta $P$ and $P^{\prime}$, and $M$ the nucleon mass). Another relevant kinematical variable is the so called skewedness, $\xi=-\Delta^{+} /\left(P^{+}+P^{\prime+}\right)^{1}$.

The measurement of GPDs is a hard experimental challenge; despite of this, data for proton and nuclear targets are being analyzed, see, e.g., Refs. [3, 4].

In particular, the study of nuclear GPDs is a relevant task to obtain new information on possible modifications of the bound nucleon structure (this analysis started in Ref. [5]), and also to extract neutron GPDs, necessary to get, together with the proton ones, a flavor decomposition of GPDs. For this kind of studies, ${ }^{3} \mathrm{He}$ is a very important target due to its spin structure (see, e.g., Refs. [6, 7]). In fact, among the

\footnotetext{
ae-mail: matteo.rinaldi@pg.infn.it

be-mail: sergio.scopetta@pg.infn.it

${ }^{1}$ In this paper, $a^{ \pm}=\left(a^{0} \pm a^{3}\right) / \sqrt{2}$.
}

This is an Open Access article distributed under the terms of the Creative Commons Attribution License 4.0, which permits unrestricted use, distribution, and reproduction in any medium, provided the original work is properly cited. 

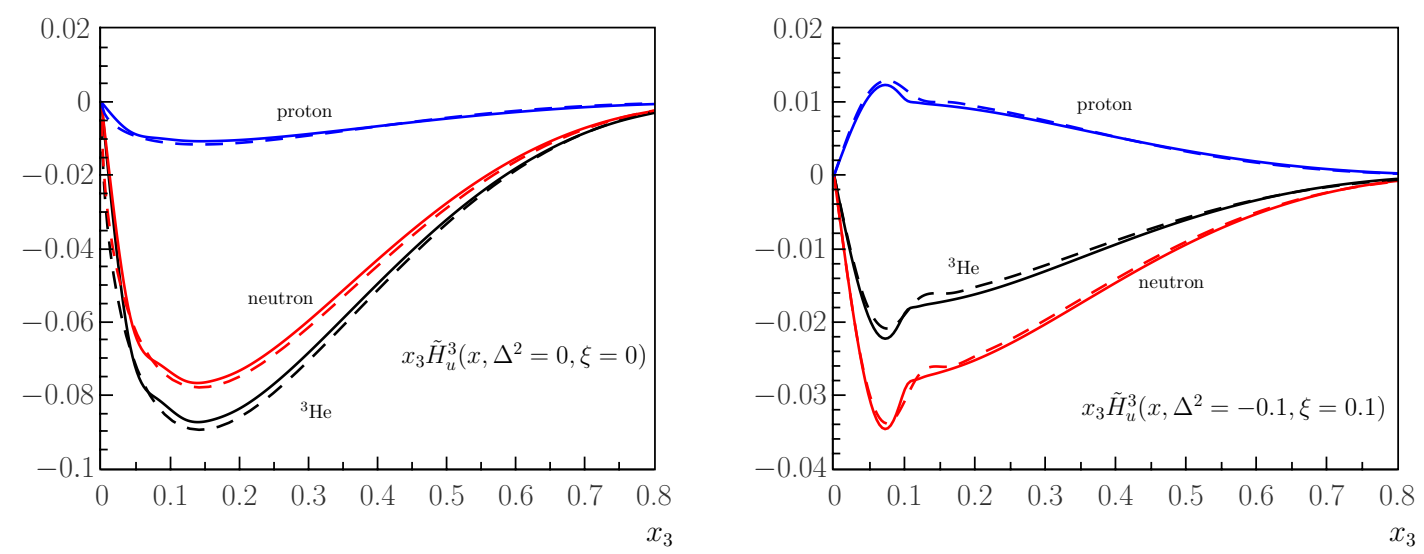

Figure 1. The GPD $x_{3} \tilde{H}^{3, u}\left(x, \Delta^{2}, \xi\right)$ for the flavor $q=u$, where $x_{3}=\left(M_{3} / M\right) x$ and $\xi_{3}=\left(M_{3} / M\right) \xi$, shown in the forward limit (left panel) and at $\Delta^{2}=-0.1 \mathrm{GeV}^{2}$ and $\xi_{3}=0.1$ (right panel), together with the neutron and the proton contribution. Solid lines represent the full IA result, Eq. (2), while the dashed ones correspond to the approximation Eq. (6).

light nuclei, ${ }^{3} \mathrm{He}$ is the only target for which the sum $\tilde{G}_{M}^{3, q}\left(x, \Delta^{2}, \xi\right)=H_{q}^{3}\left(x, \Delta^{2}, \xi\right)+E_{q}^{3}\left(x, \Delta^{2}, \xi\right)$ could be dominated by the neutron contribution. In facts, ${ }^{2} \mathrm{H}$ and ${ }^{4} \mathrm{He}$ are isoscalar and not useful to this aim, see Ref. [8]. In this latter work it has been also shown to what extent this fact can be used to extract the neutron information.

The details of the calculation of ${ }^{3} \mathrm{He}$ GPDs in IA can be found in Ref. [9], where the GPD $H$ of ${ }^{3} \mathrm{He}$, $H_{q}^{3}$, has been obtained as a convolution-like equation in terms of the corresponding nucleon quantity. The treatment has been later extended to $\tilde{G}_{M}^{3, q}$ (see Ref. [8] for details), and to $\tilde{H}_{q}^{3}$, giving the following result:

and

$$
\tilde{G}_{M}^{3, q}\left(x, \Delta^{2}, \xi\right)=\sum_{N} \int d E \int d \vec{p}\left[P_{+-,+-}^{N}-P_{+-,-+}^{N}\right]\left(\vec{p}, \vec{p}^{\prime}, E\right) \frac{\xi^{\prime}}{\xi} \tilde{G}_{M}^{N, q}\left(x^{\prime}, \Delta^{2}, \xi^{\prime}\right),
$$

respectively.

$$
\tilde{H}_{q}^{3}\left(x, \Delta^{2}, \xi\right)=\sum_{N} \int d E \int d \vec{p}\left[P_{++,++}^{N}-P_{++,--}^{N}\right]\left(\vec{p}, \vec{p}^{\prime}, E\right) \frac{\xi^{\prime}}{\xi} \tilde{H}_{q}^{N}\left(x^{\prime}, \Delta^{2}, \xi^{\prime}\right),
$$

Here, $x^{\prime}$ and $\xi^{\prime}$ are the variables for the bound nucleon GPDs, where $p\left(p^{\prime}=p+\Delta\right)$ is its 4-momentum in the initial (final) state and, eventually, proper components appear of the spin dependent, one body off diagonal spectral function:

$$
P_{S S^{\prime}, s s^{\prime}}^{N}\left(\vec{p}, \vec{p}^{\prime}, E\right)=\frac{1}{(2 \pi)^{6}} \frac{M \sqrt{M E}}{2} \int d \Omega_{t} \sum_{s_{t}}\left\langle\vec{P}^{\prime} S^{\prime} \mid \vec{p}^{\prime} s^{\prime}, \vec{t} s_{t}\right\rangle_{N}\left\langle\vec{p} s, \vec{t} s_{t} \mid \vec{P} S\right\rangle_{N},
$$

where $S, S^{\prime}\left(s, s^{\prime}\right)$ are the nuclear (nucleon) spin projections in the initial (final) state, respectively, and $E=E_{\min }+E_{R}^{*}$, being $E_{R}^{*}$ the excitation energy of the full interacting two-body recoiling system. The main quantity appearing in the definition Eq. (3) is the intrinsic overlap integral

$$
\left\langle\vec{p} s, \vec{t} s_{t} \mid \vec{P} S\right\rangle_{N}=\int d \vec{y} e^{i \vec{p} \cdot \vec{y}}\left\langle\chi_{N}^{s}, \Psi_{t}^{s_{t}}(\vec{x}) \mid \Psi_{3}^{S}(\vec{x}, \vec{y})\right\rangle
$$

between the wave function of ${ }^{3} \mathrm{He}, \Psi_{3}^{S}$, and the final state, described by two wave functions: $\left.i\right) \Psi_{t}^{S_{t}}$, eigenfunction with eigenvalue $E=E_{\text {min }}+E_{R}^{*}$, of the state with intrinsic quantum numbers $s_{t}$ of the 
Hamiltonian pertaining to the system of two interacting nucleons with relative momentum $\vec{t}$, which can be either a bound or a scattering state, and ii) the plane wave representing the nucleon $N$ in IA. A numerical evaluation of Eqs. (1) and (2), requires the knowledge of the overlaps, Eq. (4), appearing in Eq. (3). Use has been made of those corresponding to the analysis presented in Ref. [10] in terms of AV18 [11] wave functions [12].

In order to numerically evaluate Eqs. ((1), (2)), A model for the free nucleonic GPDs has to be chosen. For $H$ and $E$ use was made of a simple model, that of Ref. [13]), later minimally extended to evaluate and describe the spin dependent GPD $\tilde{H}$ (see Ref. [8] for details). In order to confirm the validity of the calculation, since data for ${ }^{3} \mathrm{He}$ GPDs are not available, it is necessary to verify the usual limits of the GPDs, i.e., the forward limit and the first moment.

In Ref. [9] it was shown that the calculation of $H_{q}^{3}\left(x, \Delta^{2}, \xi\right)$ fulfills these constraints, while for $\tilde{G}_{q}^{3}\left(x, \Delta^{2}, \xi\right)$, since the forward limit of the GPD $E$ is not defined, the only available check is the first moment. In particular, we have calculated $\sum_{q} \int d x \tilde{G}_{M}^{3, q}\left(x, \Delta^{2}, \xi\right)=G_{M}^{3}\left(\Delta^{2}\right)$, which is the well known magnetic form factor (ff) of ${ }^{3} \mathrm{He}$. Our result is found to be in perfect agreement with previous calculations (e.g. the one-body part of the AV18 calculation presented in Ref. [14]) and, for $-\Delta^{2} \leq$ $0.15 \mathrm{GeV}^{2}$, which is the relevant kinematic region for the coherent DVCS at JLab, our results compare well also with the data. In the $\tilde{H}_{q}^{3}$ case, we found that our calculation, in the forward limit, reproduces formally and numerically the results of Ref. [7] for polarized DIS off ${ }^{3} \mathrm{He}$. Anyway it is necessary to remark that the first moment of $\tilde{H}_{q}^{3}$ is related to the axial ff off ${ }^{3} \mathrm{He}$, which is poorly known, so that this check is not really useful for comparison. After this positive check, we analyze next the proton and neutron contribution to ${ }^{3} \mathrm{He}$ GPDs. In particular, $\tilde{H}_{q}^{3}$, related to a polarized target, should be dominated by the neutron contribution. Let us show now to what extent this feature is obtained and how, thanks to this observation, the neutron information can be extracted. In Fig. 1 are shown numerical results of Eq. (2) for two different values of momentum transfer. The neutron contribution is found to largely dominate the ${ }^{3} \mathrm{He}$ GPD, but increasing $\Delta^{2}$ the proton contribution grows up (see Fig. 1, solid lines in both panels), in particular for $u$ flavor. Therefore, it is necessary to introduce a procedure to safely extract the neutron information from ${ }^{3} \mathrm{He}$ data. This can be obtained by noticing that that Eq. (2) can be written:

$$
\tilde{H}_{q}^{3}\left(x, \Delta^{2}, \xi\right)=\sum_{N} \int_{x_{3}}^{\frac{M_{A}}{M}} \frac{d z}{z} h_{N}^{3}\left(z, \Delta^{2}, \xi\right) \tilde{H}_{q}^{N}\left(\frac{x}{z}, \Delta^{2}, \frac{\xi}{z},\right),
$$

where $h_{N}^{3}\left(z, \Delta^{2}, \xi\right)$ is a "light cone spin dependent off-forward momentum distribution" which is strongly peaked around $z=1$ close to the forward limit. Therefore, in this region, for $x_{3}=\left(M_{A} / M\right) x \leq$ 0.7 one has:

$$
\begin{aligned}
\tilde{H}_{q}^{3}\left(x, \Delta^{2}, \xi\right) & \simeq \sum_{N} \tilde{H}_{q}^{N}\left(x, \Delta^{2}, \xi\right) \int_{0}^{\frac{M_{A}}{M}} d z h_{N}^{3}\left(z, \Delta^{2}, \xi\right) \\
& =G_{A}^{3, p, p o i n t}\left(\Delta^{2}\right) \tilde{H}_{q}^{p}\left(x, \Delta^{2}, \xi\right)+G_{A}^{3, n, p o i n t}\left(\Delta^{2}\right) \tilde{H}_{q}^{n}\left(x, \Delta^{2}, \xi\right)
\end{aligned}
$$

Here, the axial point like ff, $G_{A}^{3, N, p o i n t}\left(\Delta^{2}\right)=\int_{0}^{\frac{M_{A}}{M}} d z h_{N}^{3}\left(z, \Delta^{2}, \xi\right)$, which would give the nuclear axial ff if the proton and the neutron were point-like systems, have been introduced.

For small values of $\Delta^{2}$ these quantities depend slowly on the nuclear potential, so that they are under good theoretical control. It is important to remark that, in the forward limit, they reproduce the so called "effective polarizations" of the protons $\left(p_{p}\right)$ and the neutron $\left(p_{n}\right)$ in ${ }^{3} \mathrm{He}$, whose values are rather similar if evaluated within different nucleon nucleon potentials (see Refs. $[6,7,10]$ for details). For the AV18 potential, used in this work, the values $p_{n}=0.878$ and $p_{p}=-0.023$ are obtained. Now we can arrange 
Eq. (6) to extract the neutron GPD from possible future data for proton and ${ }^{3} \mathrm{He}$ :

$$
\tilde{H}^{n, \text { extr }}\left(x, \Delta^{2}, \xi\right) \simeq \frac{1}{G_{A}^{3, \text { n,point }}\left(\Delta^{2}\right)}\left\{\tilde{H}^{3}\left(x, \Delta^{2}, \xi\right)-G_{A}^{3, p, \text { point }}\left(\Delta^{2}\right) \tilde{H}^{p}\left(x, \Delta^{2}, \xi\right)\right\} .
$$

We have compared the free neutron GPD of the used model with the numerical evaluation of Eq. (7), using the same nucleonic model for $\tilde{H}_{p}$ and the full calculation of $\tilde{H}^{3}$. Figure 1 (right panel) shows that the procedure works, as we expected, in particular for $x \leq 0.7$, which is the relevant region for DVCS at JLab. It is remarkable that the only theoretical ingredients of the calculation are the axial point like ffs. Our results confirm that coherent DVCS off ${ }^{3} \mathrm{He}$, at low momentum transfer $\Delta^{2}$, is an ideal process to obtain the neutron GPDs. If the experimental kinematic region were extended at higher $\Delta^{2}$, a relativistic treatment [15] and/or the inclusion of many body currents, beyond the present IA scheme, should be implemented. Now we have at hand all the ingredients for the evaluation of the cross section asymmetries, at leading twist, for a spin 1/2 target. This analysis is going on using the formalism introduced in Ref. [16].

This work was supported in part by the Research Infrastructure Integrating Activity Study of Strongly Interacting Matter (acronym HadronPhysic3, Grant Agreement n. 283286) under the Seventh Framework Programme of the European Community.

\section{References}

[1] D. Mueller, et al., Fortsch. Phys. 42, 101 (1994)

[2] A.V. Radyushkin, Phys. Lett. B 380, 417 (1996); X.-D. Ji, Phys. Rev. Lett. 78, 610 (1997)

[3] M. Mazouz, et al. , [Jefferson Lab Hall A Collaboration], Phys. Rev. Lett. 99, 242501 (2007)

[4] M. Guidal, Phys. Lett. B 693, 17 (2010)

[5] E.R. Berger, F. Cano, M. Diehl, and B. Pire, Phys. Rev. Lett. 87, 142302 (2001)

[6] J.L. Friar, et al., Phys. Rev. C 42, 2310 (1990)

[7] C. Ciofi degli Atti, et al., Phys. Rev. C 48, 968 (1993)

[8] M. Rinaldi, and S. Scopetta, Phys. Rev. C 85, 062201(R) (2012); M. Rinaldi, and S. Scopetta

[9] S. Scopetta, Phys. Rev. C 70, 015205 (2004); S. Scopetta, Phys. Rev. C 79, 025207 (2009)

[10] A. Kievsky, E. Pace, G. Salmè, and M. Viviani, Phys. Rev. C 56, 64 (1997)

[11] R.B. Wiringa, V.G.J. Stoks and R. Schiavilla, Phys. Rev. C 51, 38 (1995)

[12] A. Kievsky, M. Viviani, and S. Rosati, Nucl. Phys. A 577, 511 (1994)

[13] I.V. Musatov, and A.V. Radyushkin, Phys. Rev. D 61 074027, (2000)

[14] L.E. Marcucci, D.O. Riska, and R. Schiavilla, Phys. Rev. C 58, 3069 (1998)

[15] E. Pace, G. Salmè, S. Scopetta, A. Del Dotto, and M. Rinaldi, Few Body Syst. 54, 1079 (2013)

[16] A.V. Belitsky, D. Mueller, L. Niedermeier, and A. Schäfer, Nucl. Phys. B 593, 289 (2001) 